\title{
Comportement au cisaillement d'un joint rocheux naturel
}

\section{GASC-BARBIER J. GUITTARD}

ERA 01 Laboratoire régional des ponts et chaussées 1, av. du Colonel-Roche

31400 Toulouse muriel.gasc@ developpement-durable. gouv.fr

Les discontinuités jouent un rôle majeur dans la stabilité des massifs rocheux. Différentes méthodes expérimentales et numériques existent pour les étudier. A I'heure où le blue book de la Société internationale de mécanique des roches (ISRM, 2007) est réédité, s'interroger sur les procédures les plus à même de caractériser le comportement du massif rocheux et plus spécifiquement des discontinuités reste particulièrement d'actualité. Cet article présente les dispositifs expérimentaux en place au LRPC de Toulouse, décrit les procédures d'essais courantes et souligne les difficultés rencontrées dans une optique de modélisation et de dimensionnement. La démarche implique la réalisation d'essais sur discontinuités naturelles et leur représentation au moyen des lois de comportement proposées dans le logiciel de calcul UDEC.

Mots-clés : discontinuité, essais de cisaillement, système expérimental.

\section{Shear behaviour of a natural rock discontinuity}

Discontinuities are of major importance considering the stability of rock masses. Different experimental and numerical methods can be used for their study. As the ( blue book» of the International Society of Rock Mechanics (ISRM, 2007) is republished, it is particularly relevant to wonder about the best procedures to characterize the behaviour of the discontinuities of the rock mass. This paper presents the LRPC Toulouse's experimental devices and describes the current testing procedures. It also points out the difficulties encountered in the aim of modelling and design. The approach involves testing on natural discontinuities and their representation using the constitutive laws proposed in the UDEC software.

Key words: discontinuity, shear test, experimental device. 


\section{Introduction}

Les discontinuités jouent un rôle majeur dans la stabilité des massifs rocheux. Suivant leur nature, leur orientation et les modifications du terrain envisagées, que ces modifications soient naturelles (érosion due aux variations climatiques par exemple) ou bien anthropiques (augmentation de la largeur d'une route, suppression de virages dangereux, etc.), ce sont bien les discontinuités qui influeront sur la stabilité du talus créé. La stabilité de l'ensemble pourra alors être étudiée d'un point de vue de l'ingénierie avec le dimensionnement de parades à mettre en œuvre pour éviter la chute de blocs ou bien sous un angle de comportement du massif, avec une modélisation 2D ou 3D des familles de discontinuités et l'affectation de propriétés à la matrice et aux joints rocheux, ou bien encore, en combinant ces deux approches.

Différentes études ont été menées pour caractériser la morphologie du joint rocheux. Riss et al. (1998a ; 1998b), Gentier et Riss (1990), Marache et al. (2001) ont proposé une caractérisation de la rugosité du joint rocheux à partir de paramètres statistiques, ces paramètres reliant la géométrie du joint à la longueur de base des aspérités. Jiang et al. (2006) ont proposé un modèle basé sur la variance des hauteurs des aspérités. Kulatilake et al. (1995a ; 1995b), Yang et Di (2001) ont étudié la possibilité d'utiliser une dimension fractale pour fournir une mesure de la rugosité des fractures rocheuses. Plusieurs expressions de loi de comportement ont été proposées, en se basant sur l'analyse de résultats expérimentaux et suivant quelques hypothèses supplémentaires : Souley et al. (1995), Souley et Homand (1996) utilisent le critère de rupture de Amadei ; Saeb et Amadei (1992) suggèrent que la contrainte résiduelle peut être estimée par la valeur de la contrainte de cisaillement au pic pour une contrainte normale donnée. Toute une école de pensée est également fondée sur l'utilisation du critère de Barton (1973) et du paramètre JRC (Barton et Choubey, 1977).

Quelle que soit la loi de comportement finalement retenue, il reste un point incontournable, si l'on veut correctement rendre compte du comportement du massif, c'est l'étude du comportement propre de la discontinuité pour déterminer les valeurs des paramètres du modèle. Il peut alors être intéressant de s'interroger sur les types d'essais et les conditions expérimentales à utiliser. Faut-il travailler à rigidité ou à effort constant en fonction de ce que l'on cherche à représenter? Faut-il permettre ou pas le déplacement des rotules? Quelle vitesse de déplacements des épontes choisir? Un programme de recherche est actuellement en cours au Laboratoire régional des ponts et chaussées de Toulouse (LRPC) pour examiner l'influence des procédures expérimentales (chemin de contraintes) et en particulier des vitesses de cisaillement sur des joints naturels dans du marbre. A l'heure où la Société internationale de mécanique des roches publie une mise à jour de ses recommandations pour la réalisation d'essais (ISRM, 2007), on présente ici une étude réalisée sur des fractures réelles, conformément à la norme expérimentale XP P 94-424 « Cisaillement direct selon une discontinuité de roche. Essai sous un effort constant, normal à la surface de discontinuité ». Cette norme expérimentale est très proche des recommandations de l'ISRM. On a utilisé les recommandations contenues dans ces textes pour encadrer les essais réalisés. En effet, le seul cheminement de contrainte prévu par ces documents concerne un cisaillement à contrainte constante. Cet article décrit une possibilité d'interprétation de ce type d'essai, fondée sur l'utilisation du logiciel Udec (Itasca, 2004).

L'essai considéré a été effectué sur une discontinuité naturelle prélevée dans un gneiss. Cet essai entrait dans le cadre des études pour l'aménagement de la RN 20 au niveau d'Ax-les-Thermes (Ariège), où de grands déblais rocheux ont été réalisés ( $48 \mathrm{~m}$ de haut au maximum, sur un linéaire de 600 m). La discontinuité présentait à son état naturel une surface rugueuse avec quelques dépôts d'argile, les deux épontes étaient jointives.

2

\section{Matériel expérimental}

Le matériel expérimental utilisé est constitué d'une presse de type MTS System pilotée par un système Testar. Elle présente une rigidité verticale de $10^{9} \mathrm{~N} \cdot \mathrm{mm}^{-1}$, une force maximale du vérin vertical de 1,1 MN et horizontal de $225 \mathrm{kN}$, associées à des courses maximales de vérins de $100 \mathrm{~mm}$ en vertical et de $50 \mathrm{~mm}$ en horizontal.

Le système pour l'essai de cisaillement est constitué de deux demi-boîtes de dimensions intérieures : $205 \mathrm{~mm} \times 205 \mathrm{~mm} \times 159 \mathrm{~mm}$ et l'espacement, en place, entre les deux demi-boîtes est de $22 \mathrm{~mm}$. Dans cette configuration, la force maximale du vérin vertical est de $500 \mathrm{kN}$; celle du vérin horizontal : $225 \mathrm{kN}$ et sa course : $50 \mathrm{~mm}$. L'ensemble est équipé de quatre capteurs de déplacements LVDT verticaux et deux capteurs de déplacements LVDT horizontaux de course $\pm 25 \mathrm{~mm}$.

La figure 1 présente le dispositif complet : essai de cisaillement en train d'être démonté. Les deux demiboîtes sont séparées ; l'échantillon testé est encore visible dans la demi-boîte inférieure.

Le système de mise en charge verticale a pour particularité de pouvoir réaliser des essais « rotulés ). Ainsi, lors du cisaillement, la demi-boîte supérieure peut se déplacer dans toutes les directions avec un enregistrement de ses mouvements par le biais des quatre capteurs LVDT verticaux. Cette rotule est bloquée avec des cales lors de la réalisation d'un essai à contrainte normale constante (XP P 94-424, AFNOR, 1993) et les mesures des capteurs enregistrées, pour vérifier l'horizontalité du système.

Par ailleurs, le système Testar permet d'effectuer des essais suivant différents cheminements de contraintes et en particulier à rigidité constante qui devraient être préférés dans le cas de dimensionnements de tunnels, de pieux, voire de talus, étant donné que dans ces cas, le comportement du massif correspond plutôt à une déformation à rigidité constante comme l'ont montré Jiang et al. (2004), par exemple. Une procédure spécifique a ainsi été développée au LRPC de Toulouse pour de tels essais.

Afin de s'assurer des conditions exactes appliquées à l'éponte pour assurer sa fermeture avant le cisaillement, un calibrage de la rigidité du dispositif a été réalisé à l'aide d'une éprouvette en acier équipée de jauges. Les déplacements de l'ensemble presse et scellement (mortier et échantillon scellés) 


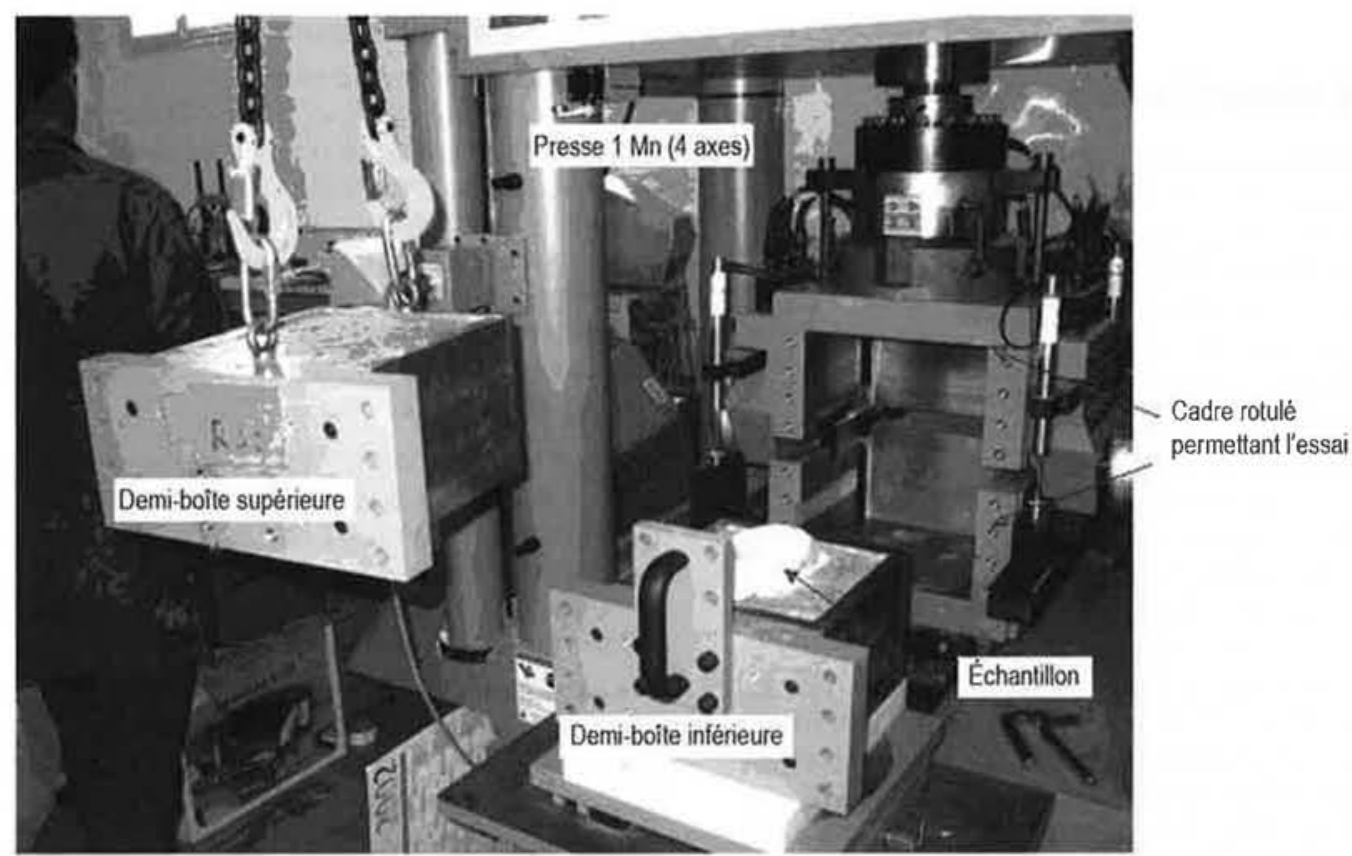

FG. 1 Photo de la presse de mécanique des roches en fin d'essai de cisaillement.

Experimental device at the end of a shear test.

sont déterminés à partir des mesures des capteurs LVDT du système corrigés par les déplacements donnés par les jauges. La figure 2 présente l'enregistrement effectué, ainsi qu'une extrapolation linéaire proposée, qui permet de rendre compte des très faibles chargements. Cette mesure donne un module de déformation du système presse et scellement de l'ordre de $57 \mathrm{MPa}$.

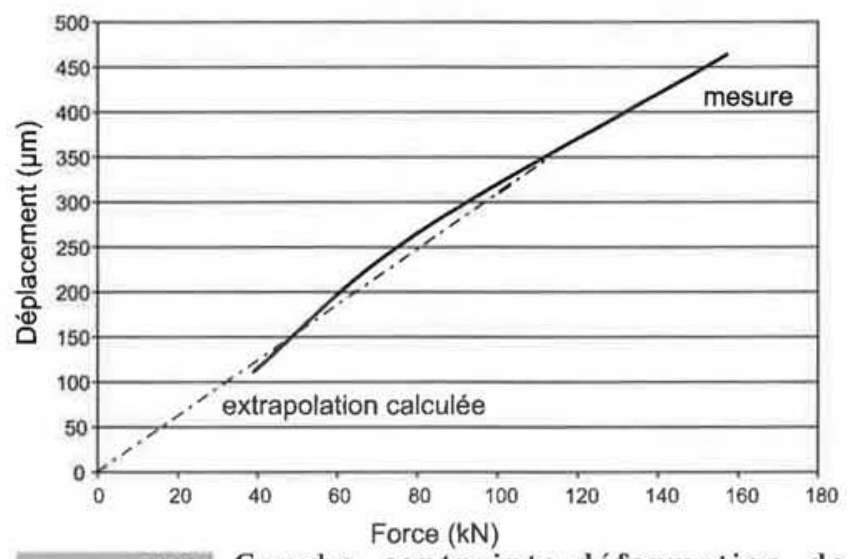

กต. 2 Courbe contrainte-déformation de l'ensemble presse + scellement.

Strain-stress curve measured and calculated for the press + cement system.

\section{Réalisation des essais de cisaillement}

Différents essais doivent être réalisés en laboratoire pour pouvoir caractériser au mieux une roche. Classiquement, pour un milieu rocheux fracturé sont réalisés des essais de caractérisation de la matrice et des essais de caractérisation des discontinuités.

\section{1}

\section{Caractérisation de la matrice}

Pour la caractérisation de la matrice sont réalisés principalement des essais uniaxiaux, conformément à la norme NF P 94-420 ou ASTM D 7012-04. Le module d'Young (E) ou module d'élasticité est usuellement défini comme la pente d'un cycle déchargement-rechargement sur la courbe contrainte-déformation, à la moitié de la contrainte de rupture. D'autres approches sont néanmoins possibles, comme par exemple celle proposée par Beniawski (1989). Le coefficient de Poisson (v) est, quant à lui, défini comme le rapport des pentes des déformations axiale et radiale. La norme française NF P 94-425 explicite plus précisément leur calcul. On obtient également la résistance à la rupture $\left(\sigma_{\mathrm{c}}\right)$.

En parallèle à ces essais de chargement, des mesures de vitesses de propagation des ondes ultrasonores sont également réalisées. Elles permettent d'obtenir des renseignements sur l'état d'altération, mais également sur la fissuration et la porosité. La norme NF P 94-411 explique leur mesure sur échantillon libre de toute contrainte. Leur estimation selon plusieurs directions peut mettre en évidence une éventuelle anisotropie de la roche. De même, la mesure intégrée des vitesses de propagation des ondes de compression et de cisaillement avant et pendant l'essai fournit des informations intéressantes sur l'évolution de la microfissuration et de l'endommagement en cours de chargement. Le dépouillement des vitesses des ondes de compression et cisaillement permet, pour chaque contrainte investiguée, de définir la valeur du module d'Young et du coefficient de Poisson par application des lois de Hooke.

La caractérisation de la matrice peut être complétée par des essais triaxiaux permettant une détermination directe de la courbe de rupture de la roche. Toutefois, ces essais, plus lourds à mettre en œuvre, restent peu pratiqués dans le cadre d'ouvrages simples, et on privilégie plutôt les méthodes empiriques. 


\section{Essais sur les discontinuités}

La majorité des problèmes d'instabilité des massifs rocheux provenant de la présence de discontinuités, il est particulièrement important de pouvoir en assurer une caractérisation fine, tant du point de vue géométrique que de leurs propriétés mécaniques.

L'échantillon, constitué de deux épontes, provient généralement d'une carotte de diamètre $90 \mathrm{~mm}$. Chaque éponte est scellée dans sa demi-boîte à l'aide de ciment. La discontinuité (naturelle ou non) à tester est placée à l'horizontale au niveau de la séparation des deux demi-boîtes (Fig. 3a). La hauteur et la forme de la carotte testée dépendent fortement de l'inclinaison de la fracture par rapport à l'axe de la carotte. Les épontes doivent présenter une surface de $63,4 \mathrm{~cm}^{2}$. Le principe de l'essai de cisaillement indiqué par la norme XP P-94-424 a également été décrit par Mazzoleni (1997). Il consiste à imposer une contrainte de cisaillement $(\tau)$ à l'échantillon, avec ou sans application d'une contrainte normale $\left(\sigma_{n}\right)$. On enregistre durant l'essai les déplacements relatifs des épontes dans la direction du cisaillement $\left(u_{t}\right)$ et dans la direction orthogonale $\left(u_{n}\right)$. Les figures $3 a$ et $3 b$ schématisent ce type d'essai et le résultat attendu.

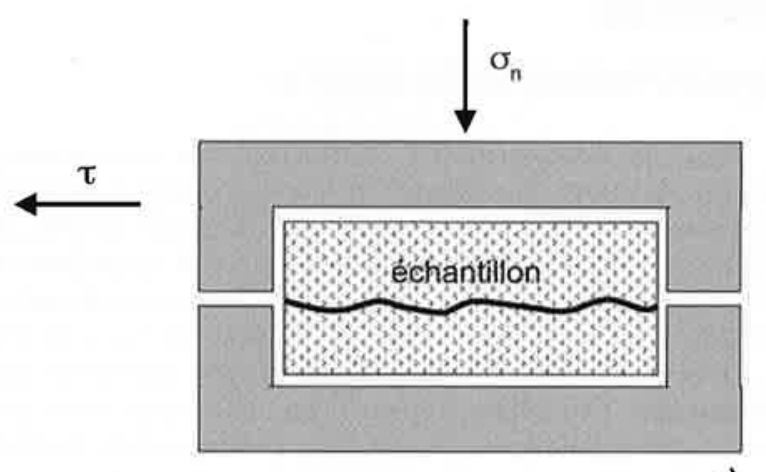

a)

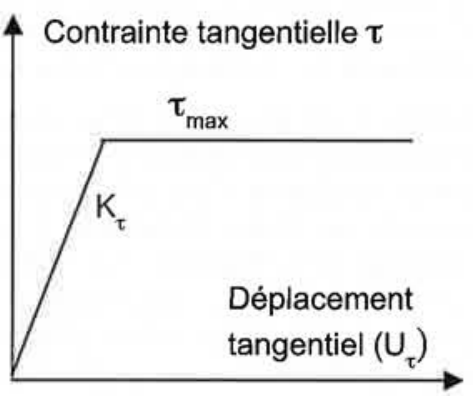

b)

FIG. 3 Principe de l'essai de cisaillement direct. a) vue en coupe de l'échantillon; b) courbe contrainte-déformation attendue.

Scheme of direct shear test. a) cross-section view ; b) shear stress vs shear displacement.

Grâce aux essais de cisaillement, on obtient les paramètres suivants.

\section{- Raideurs}

La raideur normale $K_{n}$ est définie par le rapport $\sigma_{n} / u_{n}$ pendant le chargement normal et la raideur tangentielle $\mathrm{K}_{\mathrm{t}}$, par le rapport $\tau / \mathrm{u}_{\mathrm{t}}$.
- Résistance au cisaillement

Les paramètres de résistance au cisaillement sont obtenus à partir de la courbe déplacement tangentielcontrainte normale. La résistance de pic est la contrainte de cisaillement maximale sur la courbe, correspondant au cisaillement des aspérités. On obtient également la résistance résiduelle qui caractérise le frottement des épontes en contact après la rupture des aspérités, et la dilatance, représentée par un déplacement des épontes dans la direction normale au plan des discontinuités.

\section{- Angles}

Pour chaque valeur de déplacement tangentiel, on peut calculer : l'angle de frottement global $\beta=\arctan$ (effort tangentiel/effort normal) ; l'angle de dilatance i = arctan (incrément de déplacement normal/incrément de déplacement tangentiel correspondant) et l'angle de frottement interne $\varphi=\beta+\mathrm{i}$.

On utilise en général un critère de Coulomb pour décrire la rupture d'une discontinuité naturelle et sa résistance résiduelle dans un plan contrainte de cisaillement maximale - contrainte normale.

L'essai de cisaillement présenté sur la figure 4 a été réalisé conformément à la norme XP P 94-424, à ceci près que la norme ne parle que du cisaillement direct, et que, dans cette étude, on a fait le choix d'effectuer un cycle complet de cisaillement. Cela signifie qu'à partir du joint en place, le cisaillement a été poursuivi jusqu'à $15 \mathrm{~mm}$, puis on a imposé la même vitesse de cisaillement mais en sens inverse jusqu'à $15 \mathrm{~mm}$, avant de revenir à la position initiale. La vitesse de cisaillement imposée pour cet essai était de $5 \mu \mathrm{m} / \mathrm{s}$. La contrainte normale constante était fixée à $2 \mathrm{MPa}$.

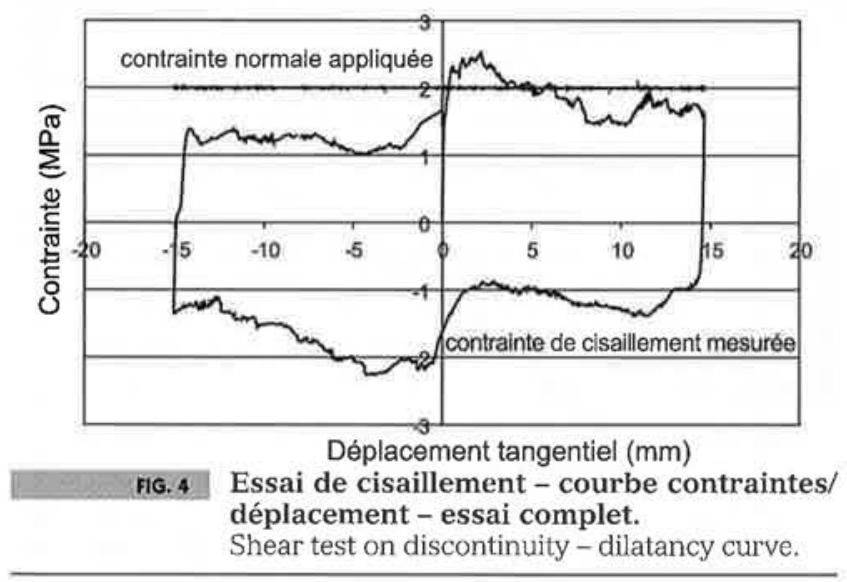

\section{3}

\section{Limites de la norme NF XP P 94-424}

Si elle traite bien du déroulement d'un essai isolé à contrainte normale constante, sa portée est en revanche limitée lorsqu'on s'intéresse à un problème de dimensionnement. En effet :

- la contrainte normale constante est le seul cas de chargement étudié :

- le traitement d'une série d'essais sous plusieurs contraintes n'est pas abordé. La mise en relation des différents résultats est pourtant un point clé si l'on souhaite caractériser le massif dans sa globalité ;

- l'essai est réalisé sur une discontinuité naturelle dont la section est de l'ordre du décimètre carré. Par conséquent, à l'échelle d'un massif, il s'agit d'un compor- 
tement très local : les ondulations d'ensemble d'une structure ne sont pas prises en compte ;

- il est souvent difficile de reproduire les conditions hydriques prévalant in situ;

- le cisaillement à contrainte normale constante est rare dans la plupart des modélisations d'un massif rocheux.

L'expression des résultats, telle que demandée par la norme, se veut indépendante de toute interprétation. Ainsi, les résultats doivent être présentés en fonction d'efforts plutôt qu'en termes de contraintes (mis à part l'évaluation de la contrainte normale) et elle définit $\varphi, \beta$ et i sans pour autant leur attribuer de signification. On considère que $\varphi$ est l'angle de frottement de la droite de Mohr-Coulomb, $\beta$ l'angle de frottement global, défini par arctan (T/N) et i l'angle de dilatance, défini par $\arctan \left(\Delta \mathrm{u}_{\mathrm{n}} / \Delta \mathrm{u}_{\mathrm{p}}\right)$.

Sur ces points, la norme française est très différente de la norme américaine ASTM D 5607-02, qui présente les caractéristiques suivantes :

- les résultats sont exprimés en termes de contraintes normale et tangentielle ( $\sigma$ et $\tau$ ) ;

- le pic est plutôt défini comme le passage entre deux phases distinctes ;

- l'angle de frottement global ( $\beta$ ) est explicitement défini comme la composition de la participation au frottement pur, la participation à la dilatance et la participation au cisaillement à travers les aspérités.

Enfin, la norme américaine fournit également des indications pour l'exploitation des résultats d'une série d'essais, concernant la représentation de la courbe de rupture de pic et de la courbe de frottement résiduel dans le plan $(\sigma-\tau)$.

Notons que les normes sont appliquées afin de disposer d'un référentiel technique commun avec les autres expérimentateurs et des matériels de capacités équivalentes. D'autres essais de cisaillement avec des cheminements de contrainte non conventionnels peuvent bien entendus être réalisés pour rendre compte de certains phénomènes (à titre d'exemple, le LRPC est ISO 17025-Cofrac pour le processus standard et un cheminement à rigidité constante).

\section{4}

\section{Proposition d'interprétation de l'essai de cisaillement en vue du dimensionnement}

L'approche présentée ici est essentiellement expérimentale et ne vise pas l'élaboration d'un nouveau modèle mécanique de comportement des joints. Dans une optique de dimensionnement, on a choisi de travailler sur des lois connues et on a essayé d'en tirer parti au mieux en fonction des résultats d'essai. Cette approche a été développée à l'aide du logiciel de calcul numérique UDEC.

\section{1}

\section{Loi de comportement pour le massif rocheux}

Le logiciel de calcul UDEC est fondé sur la méthode des éléments distincts, mise au point par Cundall (1971, 1980 ; Cundall et Hart, 1988 ; Hart et al., 1988 ; Starfield et Cundall, 1988). Cette méthode se distingue par trois caractéristiques fondamentales :

- le massif rocheux fracturé est représenté sous la forme d'un milieu discontinu, constitué par un assemblage de blocs rigides ou déformables qui interagissent par contact de leurs angles et de leurs côtés, grâce à des joints déformables, au niveau desquels de grands déplacements, des rotations et des ruptures de contact sont possibles (Alfonsi et al., 1998) ;

- les discontinuités sont considérées comme des interactions entre blocs. Les forces de contact et les déplacements aux interfaces sont déterminés par la résolution des équations fondamentales de la dynamique ;

- le temps intervient de manière explicite dans la résolution des équations du mouvement. On peut ainsi simuler des comportements non linéaires de la roche et des discontinuités et traiter des problèmes dynamiques (séismes, explosions). Le pas de temps dépend de la masse du plus petit bloc et des caractéristiques mécaniques des joints.

Ce logiciel a pour avantage d'affecter des lois de comportement différentes à la matrice rocheuse et aux joints. Différents modèles de matériaux sont disponibles pour décrire le comportement de la matrice rocheuse, indépendamment des modèles de joints. Plusieurs modèles existent, essentiellement caractérisés par l'évolution du comportement après le pic, dérivés du modèle de Mohr-Coulomb, avec ou sans radoucissement. Ces modèles ne sont pas satisfaisants pour rendre compte du comportement particulier d'un joint, car ils sont beaucoup trop simplistes. On a donc choisi d'utiliser le modèle à écrouissage continu Continuous Yielding et noté par la suite CY. Ce modèle proposé par Cundall et Hart (1985) est un modèle empirique, qui permet de rendre correctement compte du comportement du joint lors d'un cisaillement. Au contraire de la plupart des autres modèles, celui-ci ne se fonde pas sur la notion de pic de rupture, et il ne distingue pas plusieurs étapes au cours du cisaillement : les lois constitutives restent les mêmes dans le domaine prépic et dans le domaine post-pic, ainsi le passage d'une phase à une autre s'effectue de manière continue. Une présentation plus complète du modèle est proposée en annexe, l'ensemble de ces modèles pourra être retrouvé dans la notice d'utilisation du logiciel UDEC (Itasca, 2004).

\section{2}

\section{Détermination des paramètres du modèle}

Le modèle Continuous Yielding est multi-paramètres et permet de rendre compte de façon satisfaisante de ce qui est observé au laboratoire. En revanche, si certains des paramètres utilisés peuvent être obtenus directement par l'exploitation des essais, d'autres ne peuvent être fournis que par la modélisation de l'essai de cisaillement.

On a donc réalisé avec le logiciel UDEC un modèle numérique simple, consistant à représenter les deux demi-épontes sous la forme de deux demi-blocs déformables et à appliquer à l'un d'eux la vitesse de déformation effectivement appliquée au cours de l'essai (soit $5 \mu \mathrm{m} / \mathrm{s}$ ), l'autre demi-bloc restant fixe. La détermination des paramètres du modèle est alors effectuée par itérations :

1) exploitation des résultats expérimentaux pour obtenir les paramètres directement mesurables (caractéristiques physiques et mécaniques) : 
2) modélisation à l'aide du logiciel UDEC du cisaillement de deux blocs sous contrainte normale constante ;

3) si la concordance n'est pas optimale avec les mesures, on fait varier les valeurs précédemment fixées, jusqu'à obtenir la superposition de la courbe expérimentale et de la courbe ( numérique » obtenue avec le modèle Continuous Yielding.

Les figures 5 et 6 présentent les comparaisons entre résultats expérimentaux et modèles obtenus pour la partie en cisaillement direct de l'essai (Fig. 4). La figure 5 compare les contraintes de cisaillement en fonction du déplacement tangentiel et la figure 6, les courbes de dilatance (déplacement normal en fonction du déplacement tangentiel).

L'exploitation de la courbe expérimentale préconisée dans la norme française permet d'obtenir les paramètres suivants :

- résistance au pic, $\tau_{\text {pic }}: 2,5 \mathrm{MPa}$;

- résistance résiduelle, $\tau_{\mathrm{r}}: 1,65 \mathrm{MPa}$;

- rigidité tangentielle initiale (moins de 0,1 $\mathrm{mm}$ de déplacement) : $13 \mathrm{MPa} / \mathrm{mm}$;

- angle de frottement global, $\beta$ : 39,5 degrés ;

- angle de frottement interne, $\varphi: 39$ degrés;

- angle de dilatance $\mathrm{i}$ : quasiment égal à zéro.

Les angles caractéristiques variant au cours de l'essai, les valeurs données correspondent aux valeurs en fin d'essai.

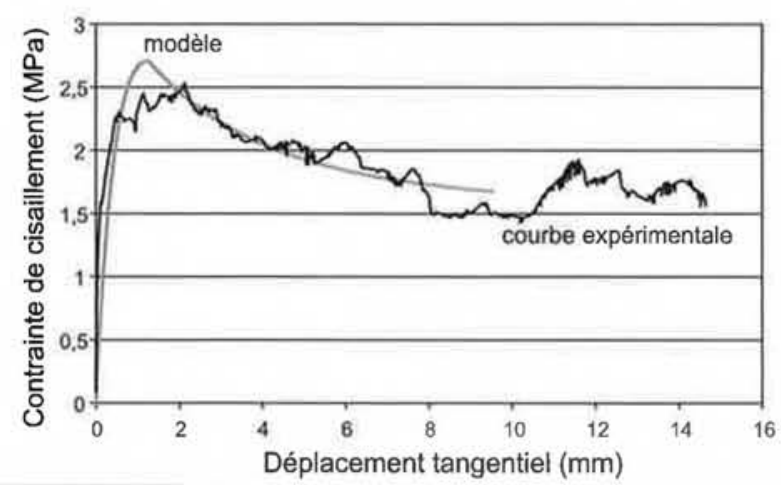

F6.5 Essai de cisaillement - courbe contrainte/ déplacement - comparaison modèle/courbe expérimentale.

Shear test on discontinuity - strain/stress curve - model and experimental curves.

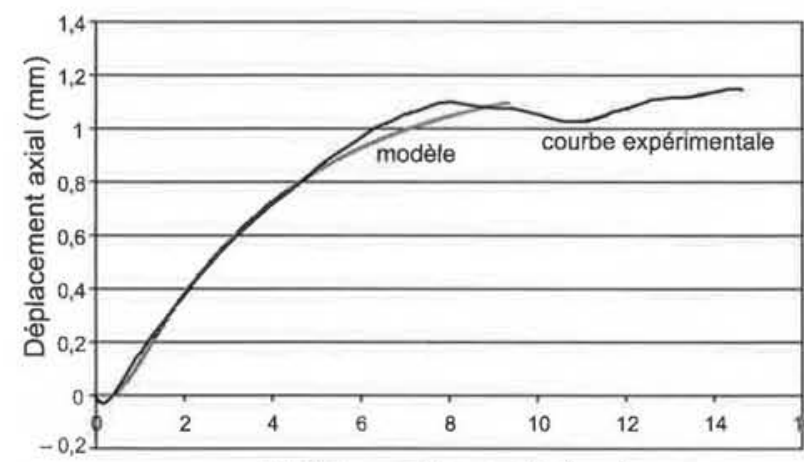

Déplacement tangentiel ( $\mathrm{mm}$ )

nG.6 Essai de cisaillement - courbe de dilatance - comparaison modèle/courbe expérimentale.

Shear test on discontinuity - dilatancy curve model and experimental curves.
La modélisation de l'essai de cisaillement à l'aide du logiciel UDEC (Figs. 5 et 6) a été réalisée à l'aide des paramètres du modèle Continuous Yielding suivants :

- angle de frottement global initial $\varphi_{\mathrm{m}}{ }^{\prime}: 57,5$ degrés ;

- angle de frottement résiduel, $\varphi_{\mathrm{r}}: 38,2$ degrés ;

- rigidité tangentielle $\mathrm{k}_{\mathrm{s}}: 7,2 \mathrm{MPa} / \mathrm{mm}$;

- paramètre de rugosite $R: 3,8 \mathrm{~mm}$.

\section{5 \\ Discussion}

Le travail présenté ici a été mené dans un cadre plus large. On a ainsi testé différentes lois de comportement disponibles dans le logiciel UDEC, pour simuler le comportement du joint rocheux, de manière à analyser l'influence de ces lois sur le comportement global d'un massif rocheux (Alfonsi et al., 2006).

La comparaison des courbes expérimentales et théoriques montre une très bonne concordance, en ce qui concerne l'évolution de la contrainte de cisaillement et de la dilatance. Les paramètres de calcul retenus sont très proches de ceux estimés sur les courbes : l'angle de frottement interne final mesuré était de 39 degrés, et l'angle retenu de 38,2 degrés. On a conservé un facteur 2 entre les valeurs de rigidité tangentielle, qui peut aisément se justifier par le fait que la courbe contrainte de cisaillement-déplacement tangentiel n'est pas linéaire, même en pré-pic (Fig. 5), et c'est donc l'ordre de grandeur de la rigidité qu'il faut prendre en compte.

Le modèle «Continuous Yielding » permet de s'affranchir des simplifications imposées par celui de Mohr-Coulomb. En particulier, il permet de modéliser le comportement au pic, puis l'obtention d'un comportement aux grandes déformations ou résiduel du joint. L'endommagement du joint et l'évolution de la dilatance sont également pris en compte.

Les résultats présentés ici montrent une bonne concordance entre la modélisation numérique et les résultats expérimentaux. Ce n'est pas toujours le cas. En effet, le même type d'exploitation des résultats a été effectué pour différentes valeurs de contrainte normale imposée et les confrontations aux mesures ne sont toujours pas aussi satisfaisantes : en particulier, et pour les mêmes matériaux, on a réalisé des cisaillements pour des contraintes normales de 0,68-5-10 et $20 \mathrm{MPa}$. A chaque fois, le comportement de la contrainte de cisaillement est relativement bien rendu par le modèle Continuous Yielding. En revanche, ce n'est pas le cas de la dilatance : pour des contraintes normales plus élevées (dans nos essais, à partir de $5 \mathrm{MPa}$ ), la dilatance mesurée n'augmente plus, mais diminue de façon significative (jusqu'à parfois devenir négative). Ce phénomène n'est pas rendu par le modèle Continuous Yielding. Ainsi, plus la contrainte normale appliquée est importante, moins on rend bien compte de la dilatance réelle de la fracture. Ce qui s'explique par le fait que la contrainte résiduelle, mesurée aux grandes déformations, est peu différente de celle trouvée au pic et, par conséquent, la simulation avec endommagement plastique est moins pertinente.

Pour des contraintes faibles, dans le cas d'épontes présentant une dilatance importante, le modèle Continuous Yielding permet de modéliser de façon satisfaisante le comportement avec pic et radoucissement observé sur la courbe contrainte de cisaillement-déplacement. 
Il est prévu, par la suite, d'étudier l'adéquation du modèle CY à des chemins de contraintes complexes, et en particulier à un cisaillement à rigidité constante.

\section{6}

\section{Conclusion}

L'étude présentée avait pour objectif d'améliorer la simulation du comportement des massifs rocheux discontinus, grâce entre autre à une meilleure évaluation des paramètres des lois de comportement utilisées pour représenter les discontinuités. Ce travail a permis de soulever la délicate question du lien quelquefois ténu entre essais de laboratoire et modélisation numérique du massif. L'ajustement itératif présenté pour les paramètres du modèle peut être sujet à caution, mais il s'appuie sur une analyse expérimentale précise et permet de rendre bien compte du comportement en laboratoire.

On a insisté sur la capacité du modèle Continuous Yielding à prendre en compte les phénomènes de pic et de radoucissement qui sont particulièrement significatifs lorsque les contraintes en jeu sont relativement faibles vis-à-vis de la résistance de la matrice.
Il est souvent délicat de passer de l'échelle de $l^{\prime}$ (c essai de laboratoire ) à l'échelle du ( massif ). Plusieurs phénomènes se cumulent, l'effet d'échelle et la représentation de la structure du massif. L'effet d'échelle peut être compris comme la généralisation des résultats d'un essai impliquant quelque décimètres carrés à une surface métrique, décamétrique ou plus, pouvant présenter en complément de la rugosité évaluée dans l'essai, des ondulations de grandes longueur d'onde. L'analyse structurale du massif doit conduire à une représentation statistiquement correcte de la répartition des discontinuités dans le massif (famille/ espacement-persistance-nature). La solution qui consiste à modéliser avec le même logiciel l'essai de laboratoire, puis de garder les mêmes paramètres pour modéliser le massif est relativement tentante (GascBarbier et al., 2008), même si elle n'est pas exempte de nouvelles questions comme celle - classique - du passage du $2 \mathrm{D}$ au $3 \mathrm{D}$.

REMERCIEMENTS

Une partie du travail présenté a été effectuée au cours du stage de M. Catillon, élève de I'ENISE. 
AFNOR - Cisaillement direct selon une discontinuité de roche. Essai sous un effort constant, normal à la surface de discontinuité. Norme XP P 94-424, 1993.

Alfonsi P., Gasc-Barbier M., Virely D. Modélisation numérique de la rupture d'une fondation sur versant rocheux : influence du modèle et des paramètres utiles pour la fracturation. ELU/ELS, 23-25 août 2006, Paris, p. 173-182.

Alfonsi P., Durville J.-L., Rachez X. - Quelques applications de la méthode des éléments distincts en mécanique des roches. BLPC, 214, 1998, p. 31-43.

Amadei B., Saeb S. - Constitutive models of rock joints. Proc. Int. Conf Rocks Joints, Loen, Norway. Barton \& Stephasson (eds). Balkema. Rotterdam. 1990, p. 581-594.

ASTM D 5607-02 - Standard test Method for Performing Direct Shear Strength Tests of Rock Specimens Under Constant Normal Force. CD-Rom annuel.

ASTM D 7012-04 - Standard test Method for Compressive Strength and Elastic Moduli of Intact Rock Core Specimens under Varying States of Stress and Temperature. CD-Rom annuel.

Barton N. - Review of a new shear strength criterion for rock joints, Eng. Geol., vol. 7, 1973.

Barton N.R., Choubey V. - The shear strength of rocks joints in theory and practice. Rock. Mech., vol. 10, 1977.

Beniawski Z. - Engineering Rock Mass Classifications, Wiley, Chischester. 1989.

Cundall P.A. - A computer model for simulation progressive, large scale movements in blocky rock systems. Int. Symp. on Rock Fracture, ISRM, Nancy (France) vol. 1, 1971, paper n II.8.

Cundall P.A. - A generalized distinct element program for modelling jointed rock. Final technical report to european Research Office. US Army, Contract DAJIA37-79-C-0548. NTIS order $n^{\circ} \mathrm{AD}-\mathrm{A} 087610 / 21980$.
Cundall P.A., Hart R. - Development of generalized $2 \mathrm{D}$ and $3 \mathrm{D}$ distinct element programs for modelling jointed rock. Itasca Consulting Group. Misc. Paper SL-85-1, US Army Corps of Engineers, 1985.

Gasc-Barbier M., Ballion A., Virely D. - Design of large cuttings in jointed rock. Bull. of Engineering geology and the Environment, $n^{\circ} 67$ (2), 2008, p. $227-$ 235.

Gentier S., Riss J. - Quantitative description and modelling of joint morphology. Proc. Int. Conf Rocks Joints, Loen, Norway, Barton \& Stephasson (eds). Balkema, 1990, p. 375-382.

Hart R., Cundall P.A., Lemos J. - Formulation of a three-dimensional element model. Int. J. of Rock Mech. Min. Sci. \& Geomech. Abstr. n² 25, 1988, p. 117-126.

ISRM - The complete ISRM suggested methods for rock characterization, testing and monitoring : 1974-2006. Ulusay \& Hudson, Ankara, Turkey, 2007, 628 p.

ITASCA - UDEC User's Guide. CD-Rom, 2004.

Jiang Y., Li B., Tanabashi Y. - Estimating the relation between surface roughness and mechanical properties of rock joints. International Journal of Rock Mechanics and Mining Sciences, $n^{\circ} 43,2006$, p. $837-$ 846.

Jiang Y., Xiao J., Tanabashi Y., Mizokami T. - Development of an automated servocontrolled direct shear apparatus applying a constant normal stiffness condition. International Journal of Rock Mechanics and Mining Sciences, $\mathrm{n}^{\circ} 41$, 2004, p. 275-286.

Kulatilake P.H., Shou G., Huang T.H., Morgan R.M. - New Peak Shear Strength Criteria for Anisotropic Rock Joints. International Journal of Rock Mechanics and Mining Sciences, vol, 32, 1995a, p. 673-697.

Kulatilake P.H., Shou G., Huang T.H. - Spectral-based peak-shear-strength criterion for rock joints. ASCE Journal of Geotechnical Engineering, vol. 121, $1995 \mathrm{~b}$.
Marache A., Riss J, Gentier S., Chiles J,-P. - Geostatistical modeling of rock fracture surfaces and simulation of shear tests. $38^{\text {th }}$ US Rock mechanics symposium, DC Rocks, 7-10 July, Washington DC (USA), Balkema, 2001, p. 869-875.

Mazzoleni G. - L'essai de cisaillement de discontinuité rocheuse. Procédure et interprétation. Bulletin des laboratoires des ponts et chaussées, 211, 1997 p. 89-99.

Riss J., Gentier S., Flamand R., Archambault G. - Detailed description of the morphology of a fracture in regard of its behaviour during shearing. Proc. MJFH 3, Vienna, Austria, April 6-9, 1998a.

Riss J., Gentier S., Hopkins D. - Shear behaviour of rock joints : prediction of the damaged areas using mathematical morphology. Acta Stereol., Origina Scientific Paper, vol. 17, 1998b, p. 303 308.

Saeb S., Amadei B. - Modelling rock joints under shear and normal loading. International Journal of Rock Mechanics and Mining Sciences, vol. 29, 1992, p. 267278.

Souley M., Homand F., Amadei B. - An extension to the Saeb and Amadei constitutive model for rock joints to include cyclic loading paths. Int. $J$. of Rock Mech. Min. Sc. \& Geom. Abs., vol. 32, 1995.

Souley M., Homand F. - Stability of rock masses evaluated by UDEC with an extended Saeb-Amadei Constitutive Law. International Journal of Rock Mechanics and Mining Sciences, vol. 33, 1996.

Starfield A.M., Cundall P.A. - Approche d'une méthodologie pour la modélisation en mécanique des roches. International Journal of Rock Mechanics and Mining Sciences, $n^{\circ} 25,1988$, p. 99-106.

Yang Z.Y., Di C.C. - A directional method for directly calculating the fractal parameters of joint surface roughness. International Journal of Rock Mechanics and Mining Sciences, n³4, 2001. 
Annexe

\section{Présentation du modèle Continuous Yielding}

Ce modèle, proposé par Cundall et Hart (1984), est un modèle empirique, créé pour représenter les phénomènes intervenant lors du cisaillement $d^{\prime} u n$ joint (diminution de la rigidité tangentielle dans la phase pré-pic, puis radoucissement, évolution continue de la dilatance, etc.). Sa principale particularité est qu'il n'est pas fondé sur la notion de pic de rupture : la valeur de la contrainte tangentielle de pic n'est pas directement utilisée, contrairement à d'autres modèles. De plus, il ne distingue pas le domaine pré-pic du domaine postpic, le passage s'effectue de manière continue (Itasca, 2004).

Dans l'écriture du modèle, les déformations élastiques sont distinguées des déformations plastiques. Le pic de contrainte tangentielle est défini par la non-augmentation des déformations élastiques. Les relations, qui gèrent la répartition des déplacements tangentiels entre les déformations élastiques et plastiques, servent de variables d'ajustement.

Les différents paramètres du modèle sont les suivants :

- la contrainte normale au joint $\sigma_{n^{\prime}}$

- la rigidité tangentielle $\mathrm{K}_{\mathrm{s}^{\prime}}$

- l'angle de frottement résiduel $\varphi$

- l'angle de frottement global initial $\varphi_{\mathrm{m}}{ }^{\mathrm{i}}$, qui est un paramètre sans signification physique réelle, mais qui peut tout de même être estimé,

- un paramètre $R$, exprimant la rugosité (en $m$ ),

- un paramètre r qui n'intervient que dans le cas d'un changement du sens du cisaillement.

Les relations liant les paramètres sont écrites de façon incrémentale, en utilisant en particulier $\Delta \tau$, incrément de contrainte tangentielle, $\Delta u_{n}$, incrément du déplacement normal et $\Delta \mathrm{u}_{\mathrm{t}}$ incrément du déplacement tangentiel, lequel est séparé en $u_{t}{ }^{\mathrm{P}}$, déplacement tangentiel plastique et $\mathrm{u}_{\mathrm{t}}^{\mathrm{E}}$, déplacement tangentiel élastique.

Le modèle utilise également les variables :

- $\varphi_{\mathrm{m}}$ dont la valeur initiale est $\varphi_{\mathrm{m}}{ }^{\mathrm{i}}$ et qui tend progressivement vers $\varphi$ à la fin du cisaillement; $\varphi_{m}$ dépend de $u_{t}{ }^{P}$ et son évolution est fonction de la rugosité (R), il correspond à l'angle de friction qu'il y aurait si le joint pouvait se dilater jusqu'à l'angle de dilatation maximal, on peut donc l'estimer comme étant égal à la valeur maximale de l'angle de dilatance ;

- $\tau_{\mathrm{m}}$ qui dépend de $\sigma_{\mathrm{n}}$ et de $\varphi_{\mathrm{m}}$ cette contrainte peut être assimilée à la valeur limite de résistance au cisaillement du joint ;

- un facteur $F$ qui dépend de la valeur courante de $\tau$ et de $\tau_{\mathrm{m}}$

De plus, le modèle suppose que l'angle de dilatance est obtenu par la différence entre l'angle de frottement global et l'angle de frottement résiduel.

Le modèle fonctionne comme suit : au début du cisaillement, le déplacement tangentiel est considéré comme complètement élastique, mais à mesure que la contrainte $\tau$ s'approche de la valeur $\tau_{m}$, les déplacements tangentiels plastiques augmentent. Dans le même temps, on considère que les dégradations du joint sont causées par les déplacements plastiques et $\tau_{m}$ diminue. Au cours du cisaillement, la part des déplacements tangentiels plastiques créés augmente jusqu'à être maximale lorsque $\tau$ atteint $\tau_{\mathrm{m}}$; à cet instant, le déplacement élastique cumulé n'évolue plus, comme la contrainte tangentielle $\tau$ qui a alors atteint sa valeur de pic.

\section{1) La phase pré-pic}

Elle se caractérise par $\tau<\tau_{m}$. Les incréments de déplacements sont définis à l'aide de la fonction $F=\frac{\left(1-\tau / \tau_{m}\right)}{1-r}$

$$
\Delta u_{t}{ }^{P}=(1-F) \Delta u_{t} \text { et } \Delta u_{t}{ }^{E}=F \Delta u_{t}
$$

On a alors $\Delta \tau=\mathrm{K}_{\mathrm{s}} \Delta \mathrm{u}_{\mathrm{t}}{ }^{\mathrm{E}}$, d'où $\Delta \tau=\mathrm{FK} \mathrm{s}_{\mathrm{s}} \Delta \mathrm{u}_{\mathrm{t}}$

L'augmentation de la contrainte tangentielle par rapport à l'augmentation du déplacement tangentiel dépend donc du produit F.K $\mathrm{K}_{\mathrm{s}}$ qui peut être considéré comme une rigidité tangentielle « instantanée »). $\mathrm{K}_{\mathrm{s}}$ est assimilée à une rigidité tangentielle ( initiale » correspondant à la valeur de la rigidité tangentielle au démarrage du cisaillement.

Plus les aspérités sont petites et plus il est difficile de les dégrader. Si $\left(\varphi_{m}-\varphi\right)$ peut être considéré comme la participation de la rugosité à la résistance de cisaillement, alors la dégradation des aspérités est prise en compte par la relation:

$$
\varphi_{\mathrm{m}}=\left(\varphi_{\mathrm{m}}^{\mathrm{i}}-\varphi\right) \exp \left(-\frac{\mathrm{u}_{\mathrm{t}}^{\mathrm{P}}}{\mathrm{R}}\right)+\varphi
$$

Cette écriture permet de comprendre l'influence du paramètre $R$ : plus $R$ est petit, plus la dégradation du joint est rapide.

2) Après le pic, la contrainte tangentielle $\tau$ n'augmente plus, mais comme les déplacements tangentiels plastiques continuent d'augmenter le joint se dégrade et la résistance du joint au cisaillement $\tau_{\mathrm{m}}$ diminue. La phase de cisaillement post-pic est caractérisée par : $\tau<\tau_{\mathrm{m}}$. On observe alors des valeurs de $F$ négatives, ce qui permet de traduire une décontraction du joint. Les déplacements élastiques cumulés diminuent et d'avantage de déplacements plastiques sont créés; la contrainte tangentielle $\tau$ diminue progressivement jusqu'à $\tau_{\mathrm{m}}$

La dilatance est déterminée à partir des valeurs de la contrainte tangentielle. L'angle de dilatance i est considéré comme la part de la contrainte tangentielle $\tau$ due au chevauchement des aspérités, il est donc calculé comme :

$$
i=\operatorname{Arctan}\left(\frac{|\tau|}{\sigma_{n}}\right)-\varphi
$$

On peut alors déterminer les déplacements normaux à partir de i par : $\Delta \mathrm{u}_{\mathrm{n}}=\Delta \mathrm{u}_{\mathrm{t}}$ tan $\mathrm{i}$.

Dans cette présentation du modèle, on a considéré que $\mathrm{K}_{\mathrm{s}}$ était un paramètre constant, or généralement $K_{\mathrm{s}}$ varie avec la contrainte normale, le modèle permet de prendre en compte ce phénomène en écrivant $\mathrm{K}_{\mathrm{s}}=\mathrm{a}_{\mathrm{s}} \sigma_{\mathrm{n}} \mathrm{b}_{\mathrm{s}}$ où $\mathrm{a}_{\mathrm{s}}$ et $\mathrm{b}_{\mathrm{s}}$ sont des paramètres empiriques à introduire. De même, les déplacements normaux dus à la fermeture du joint peuvent être associés aux déplacements normaux liés au phénomène de dilatance permettant ainsi au modèle de reproduire des chemins de contraintes autres que ceux des essais proposés ici. On peut enfin noter que par défaut, la relation donnant $K_{n}$ est : $K_{n}=a_{n} \sigma_{n}{ }^{b_{n}}$. 